\title{
Apego parental y riesgo suicida en adolescentes y jóvenes ${ }^{5}$
}

\author{
Yuly Paola Suárez Colorado \\ Magister en Psicología \\ Universidad Cooperativa de Colombia, Santa Marta \\ Colombia \\ Correo electrónico: yuli.suarezcol@campusucc.edu.co
}

José David Ebratt Pedraza

Psicólogo

Universidad del Magdalena, Santa Marta

Colombia

Correo electrónico: joseebrath2.0@gmail.com

\author{
Cristian Samper Santiago \\ Psicólogo \\ Universidad del Magdalena, Santa Marta \\ Colombia \\ Correo electrónico: criss.58698@gmail.com
}

Joe Medina Cotes

Psicólogo

Universidad del Magdalena, Santa Marta

Colombia

Correo electrónico: elchicoj9@gmail.com

Recibido: $13 / 08 / 2018$

Evaluado: 24/09/2018

Aceptado: 04/10/2018

\section{Resumen}

El estudio tuvo por objetivo identificar la asociación entre el riesgo suicida y apego parental en adolescentes y jóvenes de Santa Marta (Colombia). Participaron 481 sujetos, $45.7 \%$ masculinos y $54.3 \%$ femeninos, entre 13 y 20 años ( $M=16.33$ DT = 1.48). Se utilizaron la Escala de Riesgo Suicida y el Inventario de Apego con padres. Los resultados mostraron que el $24.5 \%$ presenta alto riesgo suicida; respecto al apego con la madre, el $14.3 \%$ presentan apego ambivalente, el $7.9 \%$ apego sin clasificar y el $1.7 \%$ apego evitativo; en cuanto al estilo de apego con el padre, el $20.2 \%$ apego ambivalente, el $18.5 \%$ apego sin clasificar y el $4.2 \%$ apego evitativo. Finalmente, existe asociación entre el apego positivo con la madre $\left(X^{2}=70.468, g l=1 ; p=.000\right)$ y el padre $\left(X^{2}=33.287, g l=1 ; p\right.$ $=.000)$ con el bajo riesgo suicida. Se concluye que el Apego Parental Negativo se asocia al alto riesgo suicida y el Apego Parental Positivo al bajo riesgo suicida.

Palabras clave

Salud mental, ideación suicida, apego, adolescentes, jóvenes.

5 Para citar este artículo: Suárez-Colorado, Y., Ebratt-Pedraza, J., Samper-Santiago, C., \& Medina-Cotes, J. (2019). Apego parental y riesgo suicida en adolescentes y jóvenes. Informes Psicológicos, 19(2), pp. 67-79 http://dx.doi. org/10.18566/infpsic.v19n2a05 


\title{
Parental attachment and suicidal risk in adolescents and young people
}

\begin{abstract}
The objective of the study was to identify the association between suicide risk and parental attachment in adolescents and youth of Santa Marta (Colombia). Participants were 481 subjects, $45.7 \%$ male and $54.3 \%$ female, between 13 and 20 years old $(M=16.33 S D=1.48)$. The Suicide Risk Scale and the Attachment Inventory with parents were used. The results showed that $24.5 \%$ present a high suicide risk. Regarding attachment to the mother, $14.3 \%$ present ambivalent attachment, $7.9 \%$ unsorted attachment and $1.7 \%$ avoidant attachment. Regarding the style of attachment with the father, 20.2\% present ambivalent attachment, 18.5\% unsorted attachment and 4.2\% avoidant attachment. Finally, there is an association between positive attachment with the mother $\left(X^{2}=70.468, g l=1 ; p=\right.$ $.000)$ and the father $\left(X^{2}=33.287, g l=1 ; p=.000\right)$ with low suicide risk. It is concluded that Negative Parental Attachment is associated with high suicide risk and Positive Parental Attachment with low suicide risk.
\end{abstract}

Keywords Mental health, suicidal ideation, attachment, adolescents, young people.

\section{Apego parental e risco suicida em adolescentes e jovens}

\section{Resumo}

0 objetivo do estudo foi identificar a associação entre risco de suicídio e apego parental em adolescentes e jovens de Santa Marta (Colômbia). Os participantes foram 481 sujeitos, 45,7\% homens e 54,3\% mulheres, entre 13 e 20 anos de idade ( $M=16,33 \mathrm{DP}=1,48)$. Utilizou-se a Escala de Risco de Suicídio e o Inventário de Anexos com os pais. Os resultados mostraram que $24,5 \%$ apresentam alto risco de suicídio; quanto ao apego à mãe, 14,3\% apresentam apego ambivalente, 7,9\% apego não triado e 1,7\% apego evitativo; em termos do estilo de apego com o pai, há 20,2\% de apego ambivalente, $18,5 \%$ de apego indiferenciado e 4,2\% de apego evitativo. Finalmente, há uma associação entre apego positivo com a mãe $\left(X^{2}=70,468, g l=1 ; p=0,000\right)$ e pai $\left(X^{2}=33,287, g l=\right.$ $1 ; p=0,000$ ) com baixo risco de suicídio. Conclui-se que 0 Apego Parental Negativo está associado com alto risco de suicídio e Apego Parental Positivo com baixo risco de suicídio.

Palavras chave Saúde mental, ideação suicida, apego, adolescentes, jovens. 


\section{ntroducción}

El estudio de la salud mental en la adolescencia implica el reconocimiento de sintomatología que desemboca en la morbilidad y mortalidad temprana. Por ejemplo, la depresión es la principal causa de enfermedad - discapacidad entre adolescentes de 10-19 años; también se estima que en 2012 fallecieron 1.3 millones de adolescentes en todo el mundo $\mathrm{y}$, entre las principales causas de mortalidad mundial, se encuentran los traumatismos por accidentes, el $\mathrm{VIH} /$ sida y el suicidio (Organización Mundial de la Salud [OMS], 2014).

En una contextualización global de una problemática de salud mundial como el suicidio, es necesario señalar que al año se suicidan aproximadamente 1 millón de personas en el mundo, es decir, 16 por cada 100.000 habitantes, sin embargo, es aún más sobresaliente el incremento del $60 \%$ de casos en los últimos 45 años, especialmente en población joven. El suicido es catalogado como la tercera causa de muerte en personas de 15 a 44 años, y la segunda entre los 10-24 años (OMS, 2012).

Como se observa, es un problema de salud pública relevante, no obstante, se estima que presenta mayor importancia estadística teniendo en cuenta que las cifras excluyen las tentativas de suicidio, las cuales son 20 veces más frecuentes que los suicidios consumados (OMS, 2012). En Colombia, se registraron 19.977 casos en la última década y para el 2017 se realizaron 2.571 necropsias debidas a suicidio, lo que indica un promedio de 5.72 casos por cada 100.000 habitantes. La tasa nacional para el rango entre 1517 años se encontró en 6.14 por cada 100.000 habitantes (Instituto de Medicina Legal y Ciencias Forensis, 2018).

Entre los factores asociados en los casos nacionales se encuentran los conflictos interpersonales, rupturas afectivas, enfermedad física-mental, muerte de un ser querido, dificultades escolares, víctima de maltrato físico-sexualpsicológico, antecedente de suicidio familiar, dificultades económicas, entre otros. Para el caso de la adolescencia, Cortés (2014) afirma que como etapa evolutiva es de vulnerabilidad y riesgo, teniendo en cuenta el aumento de demandas, inmadurez e inexperiencia que propician estados de angustia, soledad y frustración, el estado disfuncional de las familias, dificultades académicas, las condiciones económicas y desarraigo cultural, entre otras situaciones adversas. El adolescente elabora sus propios códigos de conducta, desarrolla el pensamiento formal, modifica su rol, consolida su identidad sexual, personalidad y, además de estos cambios psicológicos y sociales, el adolescente se enfrenta a cambios biológicos.

Si bien las circunstancias sociales, psicológicas, biológicas generan mayor vulnerabilidad, la explicación del por qué no todos los individuos bajo estas condiciones se precipitarían al suicidio le corresponde al campo de la psicología (Durkheim, 1987). Las diferentes teorías del desarrollo pueden explicar este tipo de comportamiento, sin embargo, es relevante un enfoque que examine factores tempranos como la teoría del apego. 
Los vínculos de apego conforman un repertorio de comportamientos que se activa en circunstancias amenazantes, estresantes o de sufrimiento con el fin de obtener apoyo, cuidado y protección del cuidador para la supervivencia (Guzmán \& Contreras, 2012). Este intercambio en el vínculo cuidador-infante es indispensable para el desarrollo de representaciones mentales acerca de la naturaleza de la relación con su figura de apego e incluso representaciones acerca de la propia existencia (Grimalt \& Heresi, 2012). El apego parental es un componente de formación en la niñez necesario para la organización de la conducta, la supervivencia de la especie, la regulación del estrés y la adaptación al medio (Bowlby, 1980). Una aproximación conceptual del apego nos conduce a la comprensión del desarrollo de la personalidad, la conducta psicopatológica y el mantenimiento de las relaciones interpersonales (González et al., 2010).

El Apego Negativo o apego inseguro han estado vinculados con sintomatología relacionada con problemas de salud mental, como el caso de los síntomas depresivos (Olhaberry et al., 2014), trastorno obsesivo-compulsivo de la personalidad (Wiltgen et al., 2015), trastorno borderline de la personalidad (Beeney, Stepp, Hallquist, Nolf, \& PiIkonis, 2015), perfeccionismo negativo (Pishva, \& Ali Besharat, 2011), ineficacia (Tavakolizadeh, Tabari \& Akbari, 2015), y recuerdos nostálgicos (Abeyta, Routledge, Roylance, \& Wildschut, 2015). Si un individuo logra un estilo de apego evitativo, tiende a tener puntos de vista más pesimistas acerca de las relaciones, de sí mismo y de otros (Feeney \& Noller, 1990), o si presenta apego ansioso-ambivalente tiende a una visión propia más negativa (Collins \& Read, 1990).

En su opuesto, el apego seguro da como resultado confianza, percepción de ambiente poco amenazante y capacidad de enfrentar los desafíos de la vida (Nolte, Guiney, Fonagy, Mayes, \& Luyten, 2011). Además, el estilo de apego seguro permite al sujeto identificar sus necesidades, buscar apoyo a favor de la diminución del malestar y mantener una representación positiva de sí mismo como de los demás (Grimalt \& Heresi, 2012).

Algunos reportes internacionales informan la relación entre el apego y diversos comportamientos suicidas: la presencia de apego inseguro materno y con pares incrementa la probabilidad de repetición de intentos de suicidio en adolescentes (Glazebrook, Townsend, \& Sayal, 2015); del mismo modo ocurre con el apego inseguro materno y paterno (Sheftall, Mathias, Furr, \& Dougherty, 2013), la ideación suicida se vincula al estilo de apego inseguro en universitarios (Ozouni, Valizadeh, \& Nikamal, 2010) e incluso a diversos trastornos mentales, además de la ideación e intentos de suicido en población general (Palitsky, Mota, Afiti, Downs, \& Sareen, 2013). Esta evidencia muestra la relevancia del apego en la psicopatología, ideación e intentos suicidas, especialmente en adolescentes y jóvenes universitarios y, ante las escasas investigaciones en contexto, este trabajo tiene por objetivo identificar la asociación entre el riesgo suicida y el apego parental en adolescentes y jóvenes de Santa Marta (Colombia). 


\section{M etodología}

\section{Diseño}

Se realizó una investigación comparativa de diseño retrospectivo, porque se analizó la relación teniendo en cuenta características diferenciales o clasificatorias presentes en los individuos, pero no manipuladas (Ato, López, \& Benavente, 2013).

\section{Participantes}

La población estuvo constituida por adolescentes escolares de secundaria y educación superior del Distrito de Santa Marta (Colombia), pertenecientes a siete instituciones educativas distritales de la comuna 6 y estudiantes de primer semestre de una universidad de la ciudad. Se realizó un muestreo probabilístico para población finita con un nivel de confianza 95\% y error estándar del 5\%; estratificación para los grados noveno, décimo y undécimo y primer semestre, para una muestra general de 582 sujetos.

La muestra definitiva fue de 481 adolescentes, con una pérdida del 17\% equivalente a 102 sujetos, por la decisión de no participar o no autorización del padre/cuidador, el olvido del consentimiento o la inasistencia a la institución en el día de aplicación. La selección se realizó aleatoriamente por el método Tómbola. Se establecieron los siguientes criterios de exclusión: no reportar una figura paterna o materna o persona que cumpla el rol de estas figuras.

Los participantes fueron $45.7 \%$ (220) masculinos y $54.3 \%$ (261) femeninos, entre 13 y 20 años (Media= 16.33; Desviación Típica=1.48).

\section{Instrumentos}

\section{Escala de Riesgo Suicida}

(Plutchik, 1989) adaptada para España por Rubio, Montero, Jáuregui, Villanueva, Casado, Marín, \& Santo Domingo (1998). El riesgo suicida se evalúa a través de 15 ítems con opción de respuesta dicotómica (si/no). La escala indaga en los intentos autolíticos previos, intensidad de la ideación actual, sentimientos de depresión, desesperanza y otros. Su calificación se obtiene al otorgar el valor de 1 a todas las respuestas afirmativas y 0 a las respuestas negativas; si la suma total de las respuestas afirmativas es superior a 6 , se considera la existencia del riesgo suicida.

El riesgo suicida indicaría la presencia de factores psicosociales que pueden llegar a precipitar comportamientos suicidas en adolescentes. La escala presenta Kuder Richardson de .89, sensibilidad y especificidad del $88 \%$ para discriminar entre individuos (Rubio et al., 1998) y en muestra colombiana fiabilidad de .84 (Aristizábal et al., 2013). La consistencia interna en este estudio se reporta en la Tabla 1. 


\section{Inventario de Apego con Padres o Pares}

(IPPA; Armsden \& Greenberg, 1987) adaptado para Colombia por Pardo, Pineda, Carrillo \& Castro (2006). Evalúa la calidad del apego percibido por los adolescentes con padres-pares mediante 25 ítems con opciones de respuesta tipo likert de Casi nunca-nunca (1) a casi siempre-siempre (5). La escala presenta un alfa de Cronbach en muestra colombiana para la subescala de la madre de .90 y para la del padre de .93 . En este estudio se excluye la subescala de pares.
Se compone de tres dimensiones: Confianza, Comunicación y Alienación. La dimensión Confianza Madre presenta alfa de Cronbach de .87, para la Comunicación Madre de .84 y Alienación Madre de .54; por su parte, la dimensión Confianza Padre presenta un alfa de Cronbach de .91, para la Comunicación Padre .88 y Alienación Padre de .68 (Pardo et al., 2006). La combinación de las subescalas establece el Apego Seguro, Apego Evitativo y Apego Ambivalente (Pardo et al., 2006).

La consistencia interna en el estudio se reporta en la Tabla 1.

Tabla 1.

Consistencia Interna de las Escalas en el Estudio

\begin{tabular}{cccccc}
\hline Dimensión & Mínimo & Máximo & Media & Desviación Típica & Alfa \\
\hline Riesgo Suicida & 0 & 14 & 3.68 & 2.97 & .76 \\
\hline Apego Madre & & & & & \\
\hline Confianza & 9 & 45 & 35.64 & 7.04 & .81 \\
Comunicación & 9 & 45 & 30.74 & 7.72 & .85 \\
Alienación & 6 & 30 & 14.31 & 4.23 & .51 \\
\hline Apego Padre & & & & & \\
\hline Confianza & 9 & 45 & 31.96 & 90.99 & .82 \\
Comunicación & 9 & 45 & 26.05 & 8.22 & .89 \\
Alienación & 6 & 30 & 14.84 & 4.72 & .59 \\
\hline
\end{tabular}

Fuente: Elaboración propia

\section{Procedimiento}

En un primer momento se solicitaron los permisos a los colegios oficiales y a una universidad de Santa Marta (Colombia); tras la aprobación de la solicitud se envió el consentimiento informado a los padres de familia en el caso de participantes menores de 18 años. Los menores de edad que devolvieron el consentimiento con la firma autorizada, diligenciaron el asentimiento informado y se les aplicó el cuadernillo. Los mayores de edad solo firmaron el consentimiento informado para 
reafirmar su participación. Las aplicaciones trascurrieron entre Octubre-Noviembre de 2016 en las aulas de clases y aulas de la Universidad, con acompañamiento de los docentes. El cuadernillo fue diligenciado con una duración entre 45 minutos y 1 hora.

\section{Análisis estadístico}

Para los datos sociodemográficos y de prevalencia se utilizó el análisis de frecuencias y estadísticos descriptivos para variables escala y ordinales. Para la asociación se dicotomizó la variable Apego Parental en Positivo y Negativo, y se utilizó el estadístico Chi Cuadrado para variables categóricas. De acuerdo con Cohen (1988), el tamaño del efecto corresponde a la magnitud en que las variables se encuentran en la población o que la hipótesis nula no se cumple. Los valores referencias para la prueba Chi Cuadrado pueden ser (.1) pequeño, (.3) mediano o (.5) grande (Cárdenas \& Arancibia, 2014). En este estudio se calculó el tamaño del efecto para la asociación entre Apego Madre / Riesgo Suicida y Apego Padre / Riesgo Suicida.

\section{Consideraciones éticas}

El estudio adoptó las consideraciones éticas para la investigación con humanos expuestos en la Ley 1090 de 2006 o Ley del Psicólogo en Colombia: secreto profesional, derecho a la no participación - retiro, consentimiento informado, devolución de resultados, anonimato. Del mismo modo, adoptó consideraciones de la Resolución 8430 de 1993 del Ministerio de Salud. El comité de ética de la Universidad estableció un riesgo mínimo para la salud de los participantes, por la sensibilidad que pueden ocasionar los ítems; empero, no se registró ningún imprevisto que afectara la integridad de adolescentes y jóvenes.

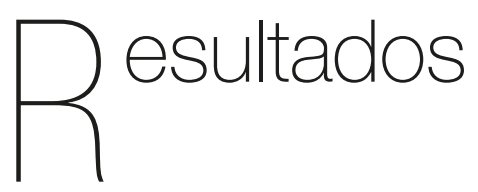

\section{Prevalencia del riesgo suicida}

El análisis muestra que un 24.5\% (118) de los adolescentes y jóvenes presentaron alto riesgo suicida (Media=3.68; Desviación Típica=2.97) y un 75.5\% (363) presentaron bajo riesgo suicida. Estos resultados evidencian una importante prevalencia del riesgo.

\section{Apego parental}

La combinación de las dimensiones Confianza, Comunicación y Alienación originan el estilo de Apego Parental. Se identificaron para Madre un 7.9\% de sujetos sin clasificar, mientras para Padre un 18.5\%. La distribución del Apego con Madre fue segura representativamente en un $76.1 \%$, así como fue segura en un 57.2\% para el Apego con Padre (ver Tabla 2). 
Tabla 2.

Apego con madre y padre en adolescentes y jóvenes

\begin{tabular}{ccc}
\hline Apego Parental & Frecuencia & Porcentaje \\
\hline Apego Madre & & \\
\hline Seguro & 366 & 76.1 \\
Ambivalente & 69 & 14.3 \\
Evitativo & 8 & 1.7 \\
Sin Clasificar & 38 & 7.9 \\
\hline Apego Padre & & \\
Seguro & 275 & 57.2 \\
Ambivalente & 97 & 20.2 \\
Evitativo & 20 & 4.2 \\
Sin Clasificar & 89 & 18.5 \\
\hline
\end{tabular}

Fuente: Elaboración propia

\section{Asociación entre el apego parental y el riesgo suicida}

Para el análisis de la asociación entre el Apego Parental y el Riesgo Suicida se recategorizaron los estilos de apego en dos categorías: Apego Positivo (Apego Seguro) y Apego Negativo (Apego
Ambivalente, Evitativo y Sin Clasificar). De este modo, se encontró, a través de la prueba chi cuadrado de Pearson, asociación entre el Apego Positivo con Madre y Padre con el Bajo Riesgo Suicida. El tamaño del efecto para la asociación del Apego Madre y Riesgo Suicida es mediano; mientras el tamaño del efecto para el Apego Padre y el Riesgo Suicida es pequeño (ver Tabla 3).

Tabla 3.

Apego con madre y padre en adolescentes y jóvenes

\begin{tabular}{ccccccc}
\hline Apego & \multicolumn{2}{c}{ Riesgo Suicida } & & Chi Cuadrado & & \multicolumn{2}{c}{$\begin{array}{c}\text { Tamaño del } \\
\text { Efecto }\end{array}$} \\
\hline & Bajo Riesgo & Alto Riesgo & $X^{2}$ & $g l$ & $p$ & $w$ \\
\hline Apego Positivo Madre & 310 & 53 & 70.468 & 1 & .000 & .3 \\
Apego Negativo Madre & 56 & 62 & & & .000 & .2 \\
Apego Positivo Padre & 233 & 130 & 33.287 & 1 & & \\
Apego Negativo Padre & 40 & 78 & & & & \\
\hline
\end{tabular}

Fuente: Elaboración propia 


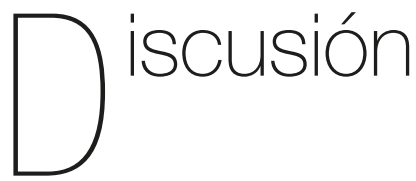

El objetivo del estudio fue identificar la asociación entre el Riesgo Suicida y Apego Parental en adolescentes y jóvenes de Santa Marta (Colombia). Se esperaba que los adolescentes con alto Riesgo Suicida presentaran mayor Apego Negativo, es decir, Evitativo o Ambivalente, en comparación con los adolescentes de bajo Riesgo Suicida (Sheftall et al., 2013).

El análisis de la asociación indicó que el Apego Parental Negativo es más característico en adolescentes con alto Riesgo Suicida. Estos resultados coinciden con los hallazgos de Glazebrook et al. (2015), quienes encontraron que los adolescentes con intentos suicidas reportaban apego negativo, así como $\mathrm{Pa}-$ litsky et al. (2013) evidenciaron que Apego Parental negativo se asociaba con intentos, ideación suicida y otros trastornos mentales. Del mismo modo, Sheftall et al. (2013) registraron en adolescentes con intentos de suicidio, menor apego materno y paterno, en comparación con adolescentes sin historial de intentos. Así mismo, un estudio de revisión indicó que el apego negativo se vincula con los comportamientos suicidas, pero afirma que no hay consensos sobre la dimensión del vínculo parental que se asocia con ideación o intentos de suicidio (Dias \& Araujo, 2016).

Como se observa, la evidencia se centra generalmente en los intentos e ideas suicidas, mientras la evaluación del riesgo suicida - conjunto de factores de riesgo de tipo psicosocial, no ha sido tan relacionado con el Apego Parental. En este sentido, el Apego Negativo coincide con la presencia de diversos factores que finalmente constituyen riesgo para el suicido.

El adolescente con Apego Negativo podría preferir la inhibición de la expresión emocional, el distanciamiento social-afectivo, bloquear recuerdos y pensamientos dolorosos, tender a la poca proximidad, suprimir emociones negativas y distanciarse de las figuras de apego; por su parte, el adolescente con estilo ambivalente podría usar algunas veces la búsqueda de proximidad, pero experimentaría hipervigilancia, rumiación y sobreactivación, además de inhibición emocional, susceptibilidad al estrés y recuerdos emocionales negativos (Garrido-Rojas, 2006). Por las anteriores características, el riesgo suicida puede ser más frecuente ante los estilos negativos o inseguros, como el apego evitativo y ambivalente

En su opuesto, la importancia del Apego Positivo ante conductas autodestructivas se centra en la posibilidad del adolescente de recurrir a la proximidad, afiliación, exploración, expresión de emociones y búsqueda de apoyo social; así mismo, el apego desde su configuración tridimensional como confianza, comunicación y menor ira u odio, potencializa el desarrollo del adolescente para ser resiliente ante las dificultades y construir una vida saludable (Calle, 2012).

De este modo, adolescentes con apego seguro podrán establecer una relación basada en la confianza y comunicación con sus padres, creando una esfera de protección antes los riesgos 
contextuales. Por demás, si los padres lograran vincularse de forma segura con sus hijos en la infancia y adolescencia, es probable que la experiencia emocional de los adolescentes y jóvenes se caracterizara mayormente por emociones positivas y una menor tendencia al afecto negativo, tristeza y apatía (Kerr, Stattin, Biesecker, \& Ferrer-Wreder, 2003; Magai et al., 2000). Del mismo modo, en la relación de Apego Positivo entre padres e hijos son fundamentales factores de crianza como una menor critica parental, padres fáciles para establecer comunicación y padres democráticos supervisores (Gómez-Ortiz, Rey, Romera, \& OrtegaRuiz, 2015).

Si estas particularidades se presentan en el vínculo, aunque se atraviese por eventos estresantes de riesgo para el suicidio, los adolescentes podrían encontrar el apoyo social y emocional necesario para afrontar el dolor (Everall et al., 2006; Shelef et al., 2015; Victor \& Klonsky, 2013).

Se recomienda nuevos estudios orientados al apego con pares y los comportamientos suicidas en adolescentes en desventaja social. Aunque el estudio presenta limitaciones por el tamaño de la muestra para jóvenes y el tamaño del efecto pequeño entre el apego con padre y riego suicida, permite una aproximación a los vínculos afectivos como protectores de conductas de riesgo para la salud.

Se concluye que el Apego Parental Positivo, es decir, el estilo de apego seguro, se asocia al bajo riesgo suicida y el Apego Parental Negativo, como el estilo ambivalente y evitativo, al alto Riesgo Suicida en adolescentes y jóvenes del estudio en Santa Marta (Colombia).

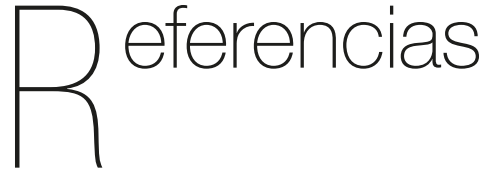

Aristizábal, C., Cañón, S., Castaño, J., Castaño, L., García, K., Marín, J.., ... \& Sánchez, G. (2013). Riesgo suicida y factores asociados en instituciones de rehabilitación para adictos a las drogas en la ciudad de Manizales (Colombia), 2012. Archivos de Medicina, 13(1), 11-23.

Abeyta, A., Routledge, C., Roylance, C., Wildschut, T., \& Sedikides, C. (2015). Attachment-related avoidance and the social and agentic content of nostalgic memories Source of the Document. Journal of Social and Personal Relationships, 32(3), 406-413. https://doi. org/10.1177/0265407514533770

Armsden, G., \& Greenberg, M. (1987). The inventory of parent and peer attachment: Individual differences and their relationship to psychological well-being in adolescence. Journal of Youth and Adolescence, 16(5), 427-454.

Ato, M., López, J., \& Benavente, A. (2013). Un sistema de clasificación de los diseños de investigación en psicología. Anales de Psicología, 29(3), 1038-1059. http://dx.doi. org/10.6018/analesps.29.3.178511

Beeney, J., Stepp, S., Hallquist, M., Nolf, K. \& Pilkonis, P. (2015). Attachment and social cognition in borderline personality disorder: Specificity in relation to antisocial and avoidant personality disorders. Personality Disorders: Theory, Research, and Treatment, 6(3), 207-215. 
Bowlby, J. (1980). Attachment and loss. New York: Basic books.

Calle, D. (2012). Apego, desarrollo y resiliencia. Informes Psicológicos, 12(1), 25-40.

Cárdenas, M. \& Arancibia, H. (2014). Potencia estadística y cálculo del tamaño del efecto en $\mathrm{G}$ * Power: complementos a las pruebas de significación estadística y su aplicación en psicología. Salud y Sociedad, 5(2), 210-224.

Collins, N., \& Read, S. (1990). Adult attachment, working models, and relationship quality in dating couples. Journal of Personality and Social Psychology, 58(4), 644-663.

Cortés, A. (2014). Conducta suicida adolescencia y riesgo. Revista Cubana Medicina General Integral, 30(1), 132-139.

Cohen, J. (1988). Statistical power analysis for the behavioral sciences (2.Ed.). Hillsdale, $\mathrm{NJ}$ : Lawrence Erlbaum.

Dias, V., \& Araujo, M. (2016). Apego e risco de suicidio em adolescents. Revista da SPAGESP, (2), 120-136.

Durkheim, E. (1897). Le suicide: étude de sociologie. París: Alcan.

Everall, R., Bostik, K., \& Paulson, B. (2006). Being in the Safety Zone Emotional Experiences of Suicidal Adolescents and Emerging Adults. Journal of Adolescent Research, 21(4), 370-392. https://doi. org/10.1177/0743558406289753

Feeney, J., \& Noller, P. (1990). Attachment style as a predictor of adult romantic relationships. Journal of personality and Social Psychology, 58(2), 281-291. https:// dx.doi.org/10.1037/0022-3514.58.2.281
Garrido-Rojas, L. (2006). Apego, emoción y regulación emocional: Implicaciones para la salud. Revista Latinoamericana de Psicología, 38(3), 493-507.

Glazebrook, K., Townsend, E., \& Sayal, K. (2015). The Role of Attachment Style in Predicting Repetition of Adolescent SelfHarm: A Longitudinal Study. Suicide and Life-Threatening Behavior, 45(6), 664-678. https://doi.org/10.1111/sltb.12159

González, R., Ysern, L., Pallás, M., Martorell, M., Marqués, C. M., \& Martín, M. (2010). Relaciones entre psicopatología y apego en la adolescencia. Revista Iberoamericana de Diagnóstico y Evaluación Psicológica, 1(29), 9-26.

Grimalt, L., \& Heresi, E. (2012). Estilos de apego y representaciones maternas durante el embarazo. Revista Chilena de Pediatría, 83(3), 239-246. http://dx.doi.org/10.4067/ S0370-41062012000300005

Gómez-Ortiz, O., Rey, R., Romera, E., \& OrtegaRuiz, R. (2015). Los estilos educativos paternos y maternos en la adolescencia y su relación con la resiliencia, el apego y la implicación en acoso escolar. Anales de Psicología, 31(3), 979-989. http://dx.doi. org/10.6018/analesps.31.3.180791

Guzmán, M., \& Contreras, P. (2012). Estilos de Apego en Relaciones de Pareja y su Asociación con la Satisfacción Marital. Psykhe(Santiago), 21(1), 69-82. http://dx.doi. org/10.4067/S0718-22282012000100005

Instituto Colombiano de medicina legal y Ciencias Forensis. (2018). Forensis. Datos para la vida 2017. Forensis, 19(1), 6-557.

Kerr, M., Stattin, H., Biesecker, G. \& FerrerWreder, L. (2003). Relationships with 
parents and peers in adolescence. En R. Lerner, M. A. Easterbrooks \& J. Mistry (Eds.), Developmental Psychology (pp. 395-419). Nueva York: Wiley

Kobak, R. R., \& Sceery, A. (1988). Attachment in late adolescence: Working models, affect regulation, and representations of self and others. Child development, 59(1), 135-146. http://dx.doi.org/10.2307/1130395

Magai, C., Hunziker, J., Mesias, W., \& Culver, L. (2000). Adult attachment styles and emotional biases. International Journal of Behavioral Development, 24(3), 301-309. https://doi. org/10.1080\%2F01650250050118286

Mikulincer, M. (1998). Adult attachment style and individual differences in functiona versus disfuntional experiences of anger. Journal of Personality and Social Pychology, 74, 513-524. http://psycnet.apa. org/doi/10.1037/0022-3514.74.2.513

Nolte, T., Guiney, J., Fonagy, P., Mayes, L., \& Luyten, P. (2011). Interpersonal stress regulation and the development of anxiety disorders: an attachment-based developmental framework. Frontiers in Behavioral Neuroscience, 5, 1-21. https:// doi.org/10.3389/fnbeh.2011.00055

Olhaberry, M., Zapata, J., Escobar, M. Mena, C., Farkas, C., Santelices, M. \& Krause, M. (2014). Antenatal depression and its relationship with problem-solving strategies, childhood abuse, social support, and attachment styles in a lowincome Chilean sample. Mental Health \& Prevention, 2(3), 86-97. https://doi. org/10.1016/j.mhp.2014.09.001

Organización Mundial de la Salud [OMS]. (2014). Primer informe de la OMS sobre prevención del suicidio. Recuperado del sitio de internet de la Organización Mundial de la Salud http://www.who. int/mediacentre/news/releases/2014/ suicide-prevention-report/es/

Organización Mundial de la Salud [OMS]. (Agosto del 2012). Prevención del suicidio (SUPRE). Recuperado del sitio de internet de la Organización Mundial de la Salud http://www.who.int/mental_health/ prevention/suicide/suicideprevent/es/.

Ozouni, R., Valizadehb, S. \& Nikam, M. (2010). The relationship between attachment styles and suicide ideation: the study of Turkmen students, Iran. Procedia Social and Behavioral Sciences, 5, 1190-1194. https:// doi.org/doi:10.1016/j.sbspro.2010.07.259

Palitsky, D., Mota, N., Afifi, T., Downs, A., \& Sareen, J. (2013). The association between adult attachment style, mental disorders, and suicidality: findings from a populationbased study. The Journal of Nervous and MentalDisease, 201(7), 579-586. https://doi. org/doi: 10.1097/NMD.0b013e31829829ab

Pardo, M., Pineda, S., Carrillo, S., \& Castro, J. (2006). Análisis psicométrico del inventario de apego con padres y pares en una muestra de adolescentes colombianos. Interamerican Journal of Psychology, 40(3), 289-302.

Pishva, N., \& Ali Besharat, M. (2011). Relationship Attachment Styles with Positive and Negative Perfectionism. Procedia - Social and Behavioral Sciences, 30, 402-406. https://doi.org/10.1016/j. sbspro.2011.10.079

Plutchik, R., \& Van Praag, H. (1989). The measurement of suicidality, aggressivity and impulsivity. Progress 
in Neuro-Psychopharmacology and Biological Psychiatry, 13, 23-34.

Rubio, G., Montero, I., Jáuregui, J., Villanueva, R., Casado, M., Marín, J. \& Santo Domingo, J. (1998). Validación de la escala de riesgo suicida de Plutchik en población española. Archivos de Neurobiología, 61(2), 143-52.

Sheftall, A., Mathias, C., Furr, R., \& Dougherty, D. (2013). Adolescent attachment security, family functioning, and suicide attempts. Attachment \& human development, 15(4), 368-383. https://doi.org/10.1080/14616734 .2013 .782649

Shelef, L., Fruchter, E., Hassidim, A., \& Zalsman, G. (2015). Emotional regulation of mental pain as moderator of suicidal ideation in military settings. European Psychiatry, 30(6), 765-769. https://doi.org/10.1016/j. eurpsy.2014.12.004
Tavakolizadeh, J., Tabari, J. \& Akbari, A. (2015). Academic Self-efficacy: Predictive Role of Attachment Styles and Meta-cognitive Skills. Procedia-Social and Behavioral Sciences, 171(16), 113-120. https://doi. org/10.1016/j.sbspro.2015.01.096

Victor, S. E., \& Klonsky, E. D. (2014). Daily emotion in non-suicidal self-injury. Journal of Clinical Psychology, 70(4), 364-375. https://doi.org/10.1002/jclp.22037

Wiltgen, A., Adler, H., Smith, R., Rufino, K., Frazier, C., Shepard, C., Booker, K., Simmons, D., Richardson, L., Allen, J. \& Fowler, C. (2015). Attachment insecurity and obsessive-compulsive personality disorder among inpatients with serious mental illness. Journal of Affective Disorders, 174, 411-415. https://doi. org/10.1016/j.jad.2014.12.011 